\title{
El coaching como herramienta estratégica para la gestión pública en la región del Cusco
}

Coaching as a strategic tool for public management in the Cusco region

Coaching como ferramenta estratégica para gestão pública na região de Cusco

\section{ARTÍCULO GENERAL}

\section{Narda Victoria Velarde Barrionuevo \\ navivelabarri@gmail.com \\ https://orcid.org/0000-0003-0387-526X}

Recibido 25 de Diciembre 2021 | Arbitrado y aceptado 25 de Diciembre 2021 | Publicado el 28 de Enero 2022

\section{RESUMEN}

El artículo se planteó llevar a cabo una exhaustiva revisión de la literatura que permita inferir que el coaching es una herramienta que puede aportar en la gestión pública a la vez que se motiva un cambio progresivo de patrones que hagan realidad la tan ansiada modernización del Estado. A través de la revisión de una serie de artículos y documentos se pudo analizar a fondo las variables involucradas tales como Coaching y Gestión Pública para analizarlas individualmente así como también inferir qué relación podía unirlas. Así, se esclareció la importancia de invertir en conocimiento en el talento humano sobre todo en el sector público. Por ello, el Coaching en el sector público debe buscar tener una formalidad que lo haga parte del desarrollo de personal y organizacional, para la reflexión de sus prácticas políticas en beneficio de la ciudadanía generado formalización de acuerdos y compromisos de la población y sus colaboradores institucionales a lograr los objetivos con políticas coordinadas y articulados para lograr la agenda propuesta para años venideros, ello sobre todo en sociedades como la peruana donde lo normal son las entidades rígidas y sin foco en el capital humano que, como se ha visto, deberían ser el centro de toda organización. Palabras clave: Coaching, herramienta estratégica, gestión pública, Cusco.

\section{ABSTRACT}

The article proposed to carry out an exhaustive review of the literature that allows inferring that coaching is a tool that can contribute to public management while motivating a progressive change of patterns that make the long-awaited modernization of the State a reality. Through the review of a series of articles and documents, it was possible to thoroughly analyze the variables involved such as Coaching and Public Management to analyze them individually as well as infer what relationship could unite them. Thus, the importance of investing in knowledge in human talent, especially in the public sector, was clarified. For this reason, Coaching in the public sector must seek to have a formality that makes it part of the personal and organizational development, for the reflection of its political practices for the benefit of citizens generated formalization of agreements and commitments of the population and its institutional collaborators. to achieve the objectives with coordinated and articulated policies to achieve the proposed agenda for years to come, especially in societies such as Peru's, where rigid entities are normal and do not focus on human capital, which, as has been seen, should be the center of any organization.

Keywords: Coaching, strategic tool, public management, Cusco.

\section{RESUMO}

O artigo se propôs a realizar uma revisão exaustiva da literatura que permite inferir que o coaching é uma ferramenta que pode contribuir para a gestão pública ao mesmo tempo em que motiva uma mudança progressiva de padrões que tornam realidade a tão esperada modernização do Estado. Por meio da revisão de uma série de artigos e documentos, foi possível analisar minuciosamente as variáveis envolvidas como Coaching e Gestão Pública para analisá-las individualmente bem como inferir qual relação poderia uni-las. Assim, ficou esclarecida a importância de investir em conhecimento no talento humano, principalmente no setor público. Por esta razão, o Coaching no setor público deve buscar ter uma formalidade que o torne parte do desenvolvimento pessoal $\mathrm{e}$ organizacional, para a reflexão de suas práticas políticas em benefício dos cidadãos gerados formalização de acordos e compromissos da população e de seus órgãos institucionais. colaboradores. alcançar os objetivos com políticas coordenadas e articuladas para alcançar a agenda proposta para os próximos anos, especialmente em sociedades como a peruana, onde entidades rígidas são normais e não se concentram no capital humano, que, como visto, deve ser o centro de qualquer organização.

Palavras-chave: Coaching, ferramenta estratégica, gestão pública, Cusco. 


\section{Introducción}

Narda Victoria Velarde Barrionuevo

El Estado peruano se compone de instituciones rígidas, la cual es una característica visible correspondiente a una gestión enfocada en tareas y resultados, más no en procesos, ni mucho menos en el desarrollo de personas, aun cuando se cuenta con la más avanzada tecnología para llevar a cabo innovaciones.

Debido a ello, se imposibilita instrumentalizar el fin supremo -la personaconsiderándola únicamente un medio. Pese a que es precisamente en los colabores, denominados servidores públicos, en quienes descansa la responsabilidad funcional y el deber moral de hacer que se geste y se redirija la empresa bajo un modelo de modernización que transforme el rol del Estado y le otorgue el llamado "rostro social" que tanto demanda la ciudadanía. Lo que a su vez generará que los gestores públicos se conviertan en verdaderos agentes de cambio, siendo reconocidos como el capital más importe, debiendo de gestionar sus competencias.

En ese sentido, la modernización de la gestión pública es un cambio que requiere ser llevado a la acción a través de un proceso de retroalimentación continuo sin obviar la planificación y organización de recursos y/o herramientas que mitiguen precisamente la resistencia al cambio, concentrando el abordaje en las personas que integran la Administración Pública.

Por tanto, se vuelve necesario profundizar en el desarrollo de las competencias individuales y grupales, destacando el fortalecimiento de los equipos de trabajo y de los liderazgos efectivos capaces de protagonizar ese cambio anhelado, en contraposición con las estructuras jerárquicas clásicas que precian habilidades técnicas y experiencia profesional por encima de las habilidades denominadas blandas. Es decir, aquellas asociadas a la personalidad y naturaleza de la persona.

Con ello planteado, en los últimos años la noción de Coaching se ha vuelto cada vez más potente, ello debido a su vinculación con el éxito en las organizaciones asociándolo a un incremento de productividad y mejoras en la gestión.

Por todo ello, la presente se plantea llevar a cabo una exhaustiva revisión de la literatura que permita inferir que el coaching es una herramienta que puede aportar en la gestión pública a la vez que se motiva un cambio progresivo de patrones que hagan realidad la tan ansiada modernización del Estado. 


\section{Metodología}

La búsqueda de la información para la revisión de literatura se hizo consultando las siguientes bases de datos como son Proquest, Scopus y Google Scholar para encontrar revistas del más alto nivel científico. Asimismo, cabe mencionar que para la realización de lo mencionado se emplearon también palabras clave como como coaching, gestión pública, herramienta estratégica.

Cada uno de estos descriptores se combinaron entre sí durante la búsqueda utilizando los operadores boléanos “and” y “or”. Además, algunos de los criterios o filtros que se utilizaron para hacer muchos más específica la búsqueda y ser más precisos con los documentos encontrados en las diferentes bases de datos consultadas fueron: que sean artículos de revistas, y la antigüedad de la publicación no mayor a 10 años, procurando que la mayor parte de las fuentes se mantenga en 5 años atrás.

Toda la información previa relacionada a los filtros aplicados y las especificaciones sirvieron como criterios de inclusión, es decir que sean artículos publicados en revistas científicas, que no excedan los diez y de preferencia cinco años de antigüedad, que la temática guarde relación con las variables de Coaching y gestión pública. Se excluyeron, en tanto, todos los documentos que estuvieran orientados a otro tipo de gestión, que no respetaran las especificaciones postuladas así como aquellos que estaban incompletos, eran más antiguos, o auqellos que tenían enlaces averiados.

\section{Resultados y discusión}

\subsection{Coaching}

\subsubsection{Nociones básicas}

De acuerdo con el origen de su terminología, la palabra Coaching se deriva del verbo inglés To Coach que significa entrenar, más el sufijo ing, que la conlleva a ser revelada en acción y significa entrenamiento. No obstante, desde otra postura en su origen lingüístico, la palabra Coach se relaciona con el termino Húngaro Kocsi, que hace referencia a un carruaje que permitía la movilidad de una o varias personas de un lugar a otro (Urgilés et al., 2019).

En el mundo empresarial se puede dilucidar que es la transición del talento humano mediante entrenamiento de un estado del conocimiento a otro orientado a cumplir con la misión y visión de la organización (Álvarez et al. 2018). 
Arroyo (2012) señala que el coaching debe entenderse como una herramienta de la gestión estratégica para las organizaciones, que puede contribuir de manera significativa en los procesos de la organización mediante un líder visionario, bajo un estilo de dirección participativo. A su vez, su resultado permitirá encontrar alternativas de solución de las variables evidenciadas que limitan la productividad y competitividad incidiendo en los resultados actuales de la entidad, de donde se deriva la importancia de un liderazgo que oriente a los colaboradores a potencializar sus competencias y empoderarlos.

Dentro del proceso de coaching, existen dos elementos claves; el Coach (primer elemento) que se encarga de optimizar las habilidades de los Coachee (segundo elemento) mediante actividades direccionadas a las áreas dentro de una organización a fin de conseguir resultados adecuados dentro de las áreas mencionadas y del entorno laboral. Ello con el fin de estar al nivel de la competitividad actual de las demás empresas, buscando obtener una ventaja que sea agente diferenciador en los servicios referentes al tema tratado en el proceso de coaching.

Según la Federación Internacional de Entrenadores también denominada Internacional Coach Federation (ICF) (2017) el Coaching supone una relación profesional entre dos personas, en la que uno es el Coach y el otro es el Coachee. La misma debe llevarse de forma continua y temporal por ambos, el coach debe conocer la temática a tratar dentro de la planificación de actividades y a su vez estar al tanto de los procesos operativos de la empresa con el objetivo de cubrir las necesidades presentadas por cada uno de los empleados presentes en los procesos mencionados.

\subsubsection{El valor del capital humano}

En las organizaciones contemporáneas del mundo tienen como pilar esencial al factor humano, una fuerza laboral capaz de crear, facilitar y aplicar conocimiento es su principal actor en la innovación y creación en manos del gerente, líder o directivo (Barrientos et al. 2020).

El valor del Capital Humano ha mantenido un auge incrementado y sostenible a partir de la década de 1980 del siglo XX, como lo registran importantes instituciones internacionales como la World Federation of People Management Associations (WFPMA), la Boston Consulting Group (BCG) y el Programa para las Naciones Unidas (PNUD). Así como también se han incorporado en muchas empresas y países de todo el 
mundo denominaciones tales como Gestión de Recursos Humanos (GRH), Gestión del Talento Humano, Gestión de Personal, Gestión Humana, entre otras (Cuesta y Valencia, 2018).

La esencia de esta nueva disciplina, de acuerdo con la fuente previa, es comprender a la persona o al ser humano en su integralidad bio-psico-social, implicando a su capacidad productiva o su capital humano. A partir de la década de 2000 de este siglo XXI, se consolida la acogida del concepto de capital humano y su gestión.

En la actualidad, la sociedad se caracteriza por estar basada en el conocimiento. Este es el principal recurso estratégico, siendo capaz por sí mismo de generar nuevo conocimiento; por ello, el conocimiento del capital humano se ha convertido en el cimiento de competitividad para cualquier empresa, puesto que su posesión confiere la posibilidad de generar una ventaja competitiva sostenible en el tiempo, e incrementa el valor de la compañía (García et al. 2012).

Debido a la rapidez con la que cambian las habilidades y conocimientos requeridos y a la consideración de los empleados como un recurso competitivo esencial para alcanzar una ventaja competitiva duradera (Guerrero y Sire, 2001), la formación se constituye en uno de los factores esenciales que determina la eficacia de las organizaciones (García et al. 2012).

En ese sentido, parte de la formación continua puede ser el coaching. De acuerdo con Pérez (2017) permite desarrollar el desempeño y para diagnosticar requiere formular y responder varias preguntas. Busca reforzar ese desempeño en los procesos en que se han identificado acciones positivas; y luego, la corrección de los comportamientos que separan al sujeto del cumplimiento de sus metas. Por lo tanto, el coach, estimula, guía y aconseja al cliente (coachee o entrenado); en varias etapas: Primero genera un contexto y un vínculo; segundo, define la situación actual; tercero, define la meta o situación ideal; cuarto, genera aprendizajes de la entrevista; quinto, desarrolla planes de acción; sexto, pasa a la acción e interioriza la responsabilidad al entrenado. Este proceso se estima entre cuatro meses y un año. (pp.20-27).

\section{El capital humano}

El capital humano se va forjando a lo largo de la historia, utilizando el conocimiento, desde las sociedades nómadas hasta nuestros días. Siendo el conocimiento el motor que ha impulsado la riqueza en las diferentes sociedades (Merzthal et al. 2017). 
Narda Victoria Velarde Barrionuevo

Varios autores como Alama (2008) citado en García et al. (2012) consideran que el capital humano está formado por los conocimientos y habilidades individuales de los miembros de la organización que permiten incrementar su productividad y su contribución a la generación de valor para la empresa. El investigador que inventa una medicina innovadora, o el gerente de planta que reduce el tiempo del ciclo de producción, son ejemplos de este tipo de capital.

El capital humano recoge la cantidad de conocimientos útiles y valiosos acumulados por los individuos en el proceso de educación y formación; incluye la capacidad y el talento innatos, así como la educación y las cualificaciones adquiridas.

\section{Invertir en el capital humano}

Los rápidos avances hacen cada vez más necesario que los trabajadores desarrollen nuevos conocimientos y nuevas habilidades. Esto es, las empresas requieren de empleados con competencias, actitudes y una agilidad mental, que les permitan pensar de manera sistémica y operar dentro de un entorno altamente dinámico (Bontis, 2002 citado en García, 2012).

Sáenz (2017) precisa que uno de los factores que se tienen en cuenta en las nuevas corrientes de la Administración moderna, es que se tengan a los colaboradores capacitados según las necesidades del negocio, invertir en el conocimiento y capacidades de estos; en la medida que esto va a repercutir en la productividad y eficiencia del personal en la institución (Bonilla et al. 2018).

Siguiendo este orden de ideas, la productividad esta enlazada a la gestión del conocimiento. Por ello, es transversal que las personas estén en constante aprendizaje y construyendo nociones por medio de diferentes sistemas (Yohalmo, 2014).

\subsubsection{El rol del coaching en el capital humano}

El desempeño del talento humano en una organización es una herramienta clave en un sistema empresarial, cuyas ventajas parten de la motivación, y de las habilidades de los gerentes, líderes y directivos. Las empresas en la actualidad requieren de un talento humano capacitado que se pueda mantener en el desempeño, a través de los principios de la confianza, sinceridad, responsabilidad y concienciación (Ravier, 2015). 
En ese sentido, el Coaching es un proceso de adiestramiento individualizado y privado, en el cual se aplican herramientas y acciones que permiten llenar el espacio entre el estado actual de un sujeto versus el estado que anhela llegar, se puede plantear de diferentes maneras, pero el diseño de un modelo técnico metodológico de coaching abarcará todo lo necesario para el correcto accionar de la empresa mediante un esquema (ASESCO, 2018).

El rol del Coaching influye de forma significativa en la productividad, como un efecto a mediano plazo, ya que en este caso se le puede proporcionar el programa a un determinado directivo, para que el resultado sea que analice la situación en la que encuentra su empresa y aprenda cómo gestionarla con otras herramientas que le permitan mejorar su productividad, a través de una mejora en su liderazgo, comunicación, toma de decisiones y demás competencias blandas y/o técnicas (Urgilés et al. 2019). Sin embargo, este proceso es siempre recomendable que se empiece por los líderes y/o directivos que son la cabeza de la institución (Yohalmo, 2014).

En relación con las actividades que realiza la gerencia ante una organización contemporánea, Barrientos et al (2020) precisa que debe atender siempre el capital humano como primer enfoque, y aunado al coaching, ello permitirá mejorar de forma permanente y tener la intención de lograr mejores resultados de valor en base al talento, sus habilidades personales y profesionales, a la actitud y a la contribución que harán desde la organización.

Pero, hay que destacar la gerencia del coaching, esta facilitará la adaptabilidad a nuevos equipos de trabajo y a nuevos cambios, con una gran capacidad para aprender en forma eficaz. Todo ello conlleva a que una organización contemporánea cuente con talento humano capacitado y preparado para el constante desarrollo y competente a enfrentar el cambio sin resistencia.

\subsubsection{Tipología del coaching}

Según quien lo recibe, los procesos de coaching se pueden recibir de forma individual, grupal o equipos, a nivel empresarial o la organización en su conjunto (Terrado, 2018).

- Coaching individual: Se basa en sesiones de aprendizaje individualizadas y personalizadas. Se trabaja en un plazo de entre 4 y 12 sesiones, durante las cuales se tratan temas de forma interactiva, con un flujo de preguntas, 
Narda Victoria Velarde Barrionuevo

herramientas, dinámicas de aprendizaje, observaciones, etc., que aportan transparencia entre coach y coachee y motiva a actuar hacia el cambio.

- Coaching de equipos: Se aplica a un equipo natural, es decir, por ejemplo, a un equipo directivo, equipo de un departamento, etc. En el cual, como indica Inglés (2013), el coach ayuda al equipo a que se vean reflejados en un espejo, es decir que vean como son como equipo, como afectan sus relaciones a los resultados y rendimiento, que tipo de roles existen en el equipo de forma informal, como suelen gestionar ciertas situaciones y los cambios.

- Coaching grupal: Consiste en la reunión de un número reducido de personas, que no constituyen un equipo natural, sino que son individuos con los mismos intereses, pero sin un objetivo común. Acompañadas de un coach, trabajan sobre un modelo de aprendizaje cooperativo a través del cual no sólo aprenden individualmente, si no que mejoran su rendimiento como grupo. Con esta herramienta los trabajadores descubren sus fortalezas y debilidades y promueve la mejora de las relaciones entre los participantes, como indica Castro (2016).

- Coaching organizacional: Este tipo de coaching actúa transformando las organizaciones, a través de la trasformación de los integrantes del misma. Se aplica a toda la organización, por tanto, en forma de coaching de equipos, grupal o individual, el coach alcanza todas y cada una de las áreas que forman la empresa.

\subsection{Gestión pública}

Pagani (2015) explica que los conceptos de gestión y gestión pública han sido muy utilizados a lo largo de las últimas décadas, principalmente a raíz del cambio del rol del Estado por el que fueron atravesando los países industrializados, y en donde se comienza a hablar de gestión como diferenciación de la administración pública tradicional. En particular el concepto de gestión se ha tomado en algunas ocasiones como sinónimo de administración, pero en otros se lo sobreentiende como un concepto superador.

Por un lado, la gestión vista como la articuladora de los recursos humanos, financieros, técnicos, organizacionales y políticos para la producción de bienes de consumo individual o colectivo que satisfagan las demandas de la sociedad, en donde se pone cierto énfasis en los problemas vinculados a la eficiencia y eficacia del conjunto de 
procesos a través de los cuales se articulan los recursos. Por otro lado, la gestión es considerada como el espacio privilegiado en donde se construye la demanda, aludiendo a los problemas políticos que tienen lugar en la dinámica de la gestión y en donde se ponen en relación el aparato estatal con la sociedad (Chiara y Di Virgilio, 2009).

Según la Secretaría de Gestión Pública en Perú, la gestión pública es un conjunto de procesos y acciones mediante los cuales las entidades tienden al logro de sus fines, objetivos y metas, a través de la gestión de políticas, recursos y programas.

\subsubsection{La Nueva Gestión Pública}

La nueva gestión pública (NGP) es una teoría que tiene como idea fuerza la adopción de una perspectiva gerencial en el Estado. Surgida en la segunda mitad del siglo $\mathrm{XX}$ en países con altos niveles de desarrollo, antagoniza con el modelo tradicional de organización y entrega de servicios públicos que se basa en ideas como la jerarquía burocrática, la planificación, la centralización y el control directo (García Moreno \& García López, 2016). En sustitución, introduce la racionalidad económica en la administración pública, como forma de aumentar la eficiencia y eficacia. Este cambio se ha concebido como un tránsito desde la administración pública a la gerencia pública.

Entre los enfoques de la NGP abordados (Chica, 2011), resaltan las nociones de reinvención del gobierno (Osborne y Gaebler, 1992), el paradigma posburocrático (Barzelay, 1998), la creación de valor público (Moore, 1995), la gestión por procesos (Aguilar, 2006) y la Gestión para resultados, GpR (BID-CLAD, 2007).

La Nueva Gestión Pública (NGP) tiene consecuencias ético- morales, al reconfigurar los valores que orientan la consecución de los fines de las organizaciones públicas y afectan la manera en que se diseña la capacidad organizacional estatal y no estatal, frente a un nuevo concepto de lo público (Chica, 2011).

\subsubsection{Gestión por procesos}

Cada actividad es una unidad de trabajo, que puede realizar un trabajo material o uno mental, y que puede ser una actividad simple o una actividad compleja que agrupa múltiples acciones más simples, llamadas "tareas" u "operaciones" (Aguilar, 2006, p. $380)$. 
Narda Victoria Velarde Barrionuevo

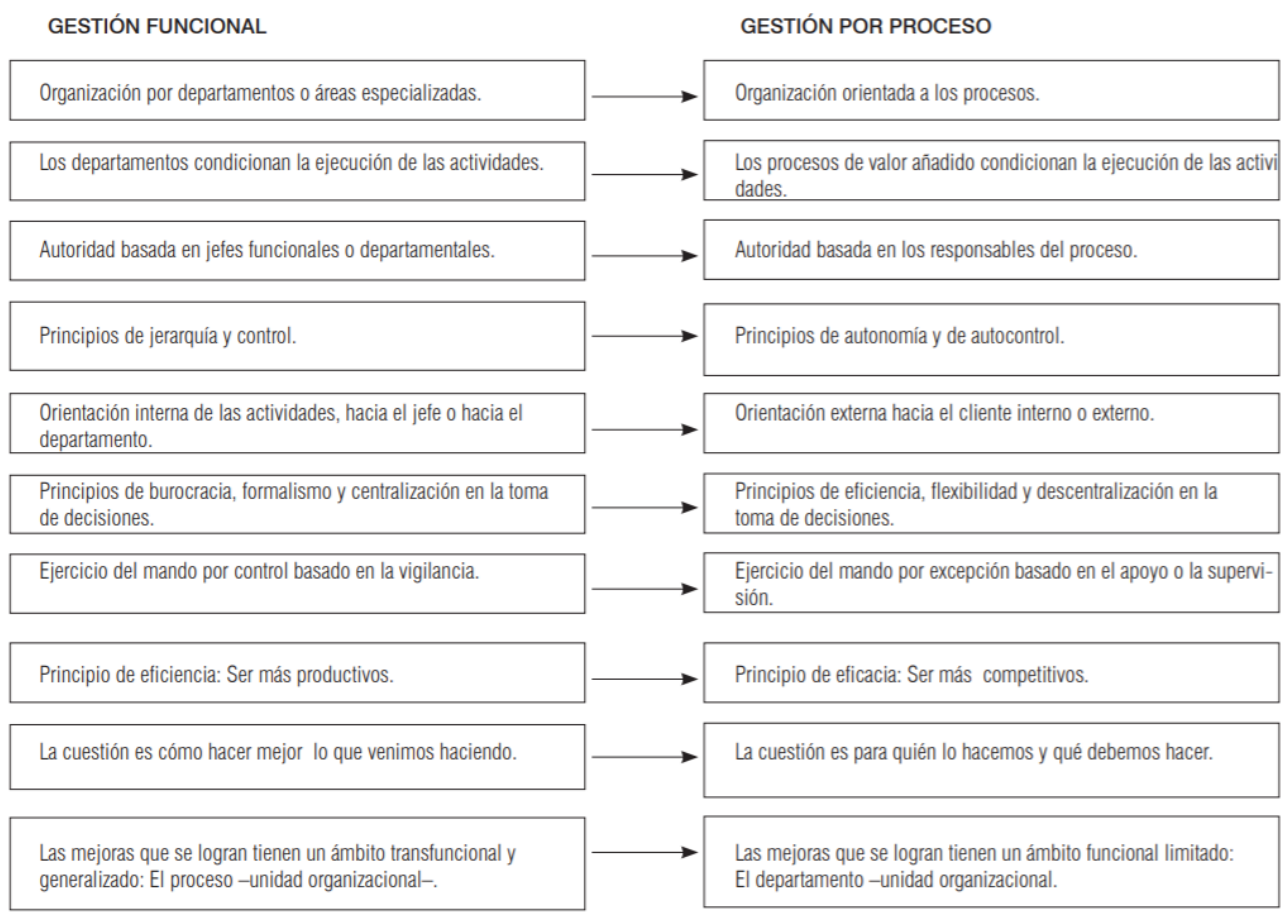

También hace énfasis en el uso de las Tecnologías de la Información y las Comunicaciones (TIC), para agilizar y permitir la realización descentralizada e incluso deslocalizada de las actividades en el marco de procesos estandarizados, en la organización de equipos multifuncionales, dado que su uso, se atribuye como garante de la disminución de tiempos y recorte de distancias en la prestación oportuna de servicios (Chica, 2011).

\subsubsection{Gestión por competencia}

La gestión por competencias, que busca aprovechar y moldear los conocimientos, habilidades, destrezas y actitudes de los individuos a fin de proporcionar el desempeño que persigue una organización competitiva

\subsubsection{Gestión por resultados}

La Gestión para Resultados (GpR), hace énfasis en la superación de problemas socialmente relevantes como: la superación de la pobreza; el crecimiento económico sostenible; el mejoramiento en la calidad de vida; el incremento de la esperanza de vida; el aumento de los promedios de escolaridad; la disminución del costo de operación gubernamental y el mejoramiento de la definición y medición de los resultados de desarrollo por parte de las instancias estatales y supraestatales.

\section{Gestión pública para resultados en el desarrollo (GpRD)}


La gestión pública para resultados en el desarrollo (GpRD), en cambio, consiste en una estrategia de gestión pensada para aumentar el nivel de desarrollo. Esta modalidad intenta lograr esto a través del uso de instrumentos de gestión que, en forma colectiva, coordinada y complementaria, orienten la acción de los actores públicos hacia la generación de mayor valor público, para generar una mayor calidad de vida de la población (García \& García, 2016).

\subsection{Coaching en gestión pública}

La modernización de la gestión pública es un cambio que requiere de un mejoramiento continuo que necesita de una planificación previa que mitigue la resistencia al cambio, centrándose en un trabajo permanente con las personas que integran la Administración Pública y, como ya se expusiera, encuentra en el Coaching una excelente herramienta de trabajo.

No sólo mitigar la resistencia al cambio es un objetivo, también lo es y de gran trascendencia, el desarrollo de las competencias individuales y grupales, destacándose el fortalecimiento de los equipos de trabajo y de los liderazgos efectivos capaces de ser agentes que destacan y revitalizan el norte modernizador, siendo protagonistas del cambio.

En consecuencia, el Coaching es una gran posibilidad de rescate y uso eficiente y efectivo de competencias grupales e individuales y por tanto de desarrollo de habilidades, capacidades, actitudes y aptitudes de todos quienes forman parte de los diversos servicios públicos.

\subsubsection{Características del coaching en el sector público}

El coaching en gestión es una iniciativa que utiliza un enfoque caracterizado por los siguientes lineamientos generales (Johnson et al. 2011).

- Generación de resultados concretos, en tiempos cortos y con mínima inversión:

El principal lineamiento es obtener resultados tangibles para la entidad pública que sean visibles en el corto plazo y que no requieran una gran inversión.

- Foco en las prioridades de la más alta autoridad institucional:

La intervención se centra en abordar las prioridades de la más alta autoridad, lo cual garantiza el compromiso y la voluntad política para realizar los 
Narda Victoria Velarde Barrionuevo

cambios requeridos y lograr los resultados esperados, permitiendo que el personal se involucre, participe y asigne el tiempo necesario para cumplir con los objetivos de la iniciativa.

- Generación de altos beneficios institucionales:

Los resultados de la intervención deben apuntar a generar beneficios institucionales y a fortalecer capacidades que excedan situaciones exclusivamente coyunturales.

- Los funcionarios de la institución son los protagonistas principales de la intervención: Una premisa fundamental es lograr que los funcionarios asuman el rol principal de agente de cambio y que el equipo BID intervenga como facilitador y "coach" del proceso. Lo anterior permite generar un ambiente de coparticipación y corresponsabilidad en la ejecución de la iniciativa, incrementando la generación y sostenibilidad de los resultados.

\subsubsection{Etapas de Coaching en el sector público}

La iniciativa se estructura en las siguientes etapas (Johnson et al. 2011):

- Primera Etapa: Planificación del Alcance

Dado el tamaño y complejidad de las instituciones, es necesario definir y lograr un acuerdo en cuanto al alcance de la intervención, es decir acotar un conjunto de resultados valiosos y alcanzables acordados con las entidades sobre las áreas de desempeño que deben ser mejoradas, así como las capacidades de gestión institucionales asociadas que requieren ser fortalecidas. Con este propósito, en conjunto con las autoridades, se realiza una evaluación general de la organización que permita identificar aquellos "puntos de dolor" prioritarios que impiden a la entidad alcanzar mejores niveles de desempeño y que, a la vez, cumplen con los lineamientos generales de la intervención.

- Segunda Etapa: Diagnóstico

Una vez que se ha determinado el alcance de la intervención, se realiza una descripción, análisis y evaluación detallada de la situación actual, que permita identificar las principales oportunidades de mejora que inciden en el desempeño de las áreas seleccionadas. Al mismo tiempo, la información 
Narda Victoria Velarde Barrionuevo

recopilada permite establecer la línea de base que medirá el nivel de logro de los resultados de la intervención.

- Tercera Etapa: Implementación

Durante esta etapa se diseña y ejecuta el plan de implementación, que comprende el conjunto de actividades necesarias para abordar las oportunidades de mejora identificadas en la etapa anterior. Esta etapa incluye también un proceso de priorización de dichas oportunidades, para seleccionar aquellas que son factibles de implementarse de forma rápida y que generen resultados concretos.

- Cuarta Etapa: Evaluación de resultados

Esta etapa contempla el seguimiento del proceso de mejora y la evaluación de los resultados obtenidos. Esta evaluación permite validar el nivel de éxito de la intervención frente a las metas esperadas.

A sabiendas que los pilares de la Gestión Pública, según Chiavenato (2012), se considera como las políticas de estado y de gobierno: el planeamiento estratégico (propósito, objetivos, misión, visión, liderazgo, estrategias políticas, procedimientos, reglas, programas, presupuestos), presupuesto por resultados, gestión de procesos, servicio civil meritocrático, seguimiento, evolución y gestión del conocimiento, es indispensable que el proceso de coaching se redirija a esto.

Por ello el coach debe guiar a los equipos a las metas, enfocarlos en los resultados, desarrollar las tareas de planificación con un diálogo concertado con la ciudadanía, la cual debe ser realizada con una comunicación asertiva, con adaptabilidad del entorno y creatividad para dar nuevas soluciones

\section{Conclusiones}

El coaching es una modalidad que va más allá de la orientación personal y de acciones ejecutivas. Pretende enfocar esfuerzos que beneficien a la empresa, los grupos y los individuos, alineando sus expectativas de desarrollo, y acompañando a los protagonistas de la organización a mejorar su desempeño y a ser productivos. De esta forma, se deduce que las acciones del coaching se relacionan con un sistema que comprenden elementos culturales y elementos relacionados con el comportamiento, las actitudes y las creencias de todos los que integran la organización. 
La información presentada invita a investigar, desarrollar y aplicar el ejercicio del coaching en los integrantes de una organización, a fin de prosperar en sus competencias $\mathrm{y}$, consecuentemente, mejorar su desempeño en el trabajo.

El Coaching en el sector público, específicamente, debe institucionalizarse como una práctica de desarrollo de personal y organizacional, para la reflexión de sus prácticas políticas en beneficio de la ciudadanía generado formalización de acuerdos $\mathrm{y}$ compromisos de la población y sus colaboradores institucionales a lograr los objetivos con políticas coordinadas y articulados para lograr la agenda propuesta para años venideros, ello sobre todo en sociedades como la peruana donde lo normal son las entidades rígidas y sin foco en el capital humano que, como se ha visto, deberían ser el centro de toda organización.

\section{Referencias}

Aguilar, L. (2006). Gobernanza y gestión pública. Sección de obras de administración pública. México: Fondo de Cultura Económica.

Alama, E. M. (2008). Capital intelectual y resultados empresariales en las empresas de servicios profesionales de España. Madrid: Universidad Complutense de Madrid.

Álvarez, L., Izquierdo, L., Gudiño, L., \& Macias, M. (2018). Coaching educativo: desarrollo de competencias en el educando de nivel superior. INNOVA Research Journal, 3(11), 169-182.

ASESCO. (6 de 2018). Asociación española de Coaching. http://www.asescoaching.org/el-coaching/

Barrientos, E., Hurtado, L., Lesmes, A. \& Duarte, D. (2020) ¿Coaching en las empresas? La gerencia del coaching en las organizaciones contemporáneas. Mundo Fesc, vol. $10, \quad$ no. $\quad$ s1, $223-236$. https://www.fesc.edu.co/Revistas/OJS/index.php/mundofesc/article/view/428/50 $\underline{4}$

Barzelay, M. (1998). Atravesando la Burocracia. Una nueva perspectiva de la Administración Pública. FCE.

Bonilla, D., Macero, R., \& Mora, E. (2018). La importancia de la capacitación en el rendimiento del personal administrativo de la Universidad Técnica de Ambato. 
Conrado,

vol.14,

no. 63 .

http://scielo.sld.cu/scielo.php?script=sci arttext\&pid=S1990-

$\underline{86442018000300268}$

Bontis, N. (2002). National capital intellectual Index: Intellectual capital development in the Arab region. Ontario: Institute for Intellectual Capital Research.

Castro, C. (2016): Coaching grupal, una metodología para potenciar talento. http://www.rrhhdigital.com/secciones/coaching/116627/Coachinggrupal-unametodologia-para-potenciar-talento-

Chiara, M. \& Di Virgilio, M. (2009). Conceptualizando la Gestión Social. En Magdalena Chiara y María Mercedes Di Virgilio (org.) La Gestión de la Política Social. Conceptos y Herramientas. Buenos Aires: Ed. Prometeo-UNGS.

Chica, S. (2011). Una mirada a los nuevos enfoques de la gestión pública. Administración \& Desarrollo, 39 (53), pp. 57-74.

Cuesta, A. \& Valencia, M. (2018) Capital Humano: Contexto de su gestión. Desafíos para $\begin{array}{llll}\text { Cuba. } & \text { Ing. } & \text { Ind } & \text { vol.39 }\end{array}$ http://scielo.sld.cu/scielo.php?script=sci arttext\&pid=S1815$\underline{59362018000200135 \# \text { B1 }}$

García, L.; García, J.; Rodríguez, A. (2012) Impacto de la inversión en capital humano sobre el valor empresarial. Academia. Revista Latinoamericana de Administración, $\quad$ núm. $\quad 51, \quad$ pp. https://www.redalyc.org/pdf/716/71625040006.pdf

García, M. \& García, R. (2016) Gestión para resultados en el ámbito público en Gestión para resultados en el desarrollo en gobiernos subnacionales, PRODEV, INDES, BID.

ICF. (04 de 2017). ICF. Obtenido de https://coachfederation.org/

Inglés, M. (2013). Maite Inglés, coach de equipos: "No hay premios especiales para los que llegan solos a la meta". http://capitalhumano.wolterskluwer.es/content/Inicio.aspx

Johnson, D., Morales, U., Pertile, G. \& Aris, D. (2011) Coaching for Results: Un enfoque innovador para fortalecer el desempeño institucional: El caso del Ministerio de 
Narda Victoria Velarde Barrionuevo

Salud Pública y Asistencia Social de la República de Guatemala. Banco Interamericano de Desarrollo.

Merzthal, J., Wakabayashi, J.L. y Talledo, H. (2017) Capital Humano y Generación de Valor en la Empresa. GECONTEC: Revista Internacional de Gestión del $\begin{array}{lllll}\text { Conocimiento } & y & \text { la }\end{array}$ https://rio.upo.es/xmlui/bitstream/handle/10433/4865/2792-8637-1PB.pdf? sequence $=1 \&$ isAllowed $=y$

Moore, M. (1995). Gestión estratégica y creación de valor en el sector público. Barcelona. España: Ediciones Paidós Ibérica.

Osborne, D. \& Gaebler, T. (1992). La Reinvención del Gobierno. New York: AddisonWesley.

Pagani, M., Payo, M. \& Galinelli, B. (2015) Estudios sobre Gestión Pública: aportes para la mejora de las organizaciones estatales en el ámbito provincial. La Plata: Subsecretaría para la Modernización del Estado; Gobierno de la provincia de Buenos Aires. http://sedici.unlp.edu.ar/handle/10915/50099

Pérez, M. (2017) Coaching. https://ebookcentral.proquest.com

Ravier, L. (2015). Principios fundamentales del coaching empresarial. International Non Directive Coaching Society. http://www.internationalcoachingsociety.com/principios-fundamentales-delcoaching-empresarial/.

Sáenz, M. (2017). Impacto de la capacitación en la mejora de la productividad en una planta de lubricantes. In Crescendo. Institucional, 8(1), pp. 93-105.

Terrados, S. (2018) El coaching en las organizaciones en el ámbito español. Universidad de Valladolid. https://uvadoc.uva.es/bitstream/handle/10324/34227/TFG-E538.pdf? sequence $=1 \&$ isAllowed $=\mathrm{y}$

Urgilés, S., Erazo, J., Narváez, C. (2019). El coaching y la productividad laboral en la Cooperativa de Ahorro y Crédito de la Pequeña Empresa Biblián Ltda. Revista Arbitrada Interdisciplinaria KOINONIA; Año IV. Vol IV. º1.

Yohalmo, R. (2014). Capacitar o morir: la capacitación de personal como estrategia competitiva (1a ed.). San Salvador: León Editores. 